\title{
Phase transition and topology in 4d simplicial gravity
}

\author{
S. Bilke ${ }^{\mathrm{a}}$, Z. Burda ${ }^{\mathrm{a} *}$, A. Krzywicki ${ }^{\mathrm{b}} \dagger$ and B. Petersson ${ }^{\mathrm{a}}$

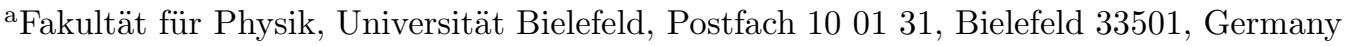 \\ bLPTHE, Bât. 211, Université de Paris-Sud, 91405 Orsay, France
}

We present data indicating that the recent evidence for the phase transition being of first order does not result from a breakdown of the ergodicity of the algorithm. We also present data showing that the thermodynamical limit of the model is independent of topology.

LPTHE Orsay 96/67, BI-TP 96/31, hep-lat/9608027

\section{Introduction}

When Euclidean Quantum Gravity is discretized following the dynamical triangulation recipe, the Einstein-Hilbert action takes the form

$S=\kappa_{4} N_{4}-\kappa_{2} N_{2}$

where $N_{i}$ is the number of $i$-simplices and $\left(\kappa_{2}, \kappa_{4}\right)$ are coupling constants. For a given topology the grand canonical partition function $Z_{G C}$ is defined by

$Z_{G C}\left(\kappa_{2}, \kappa_{4}\right)=\sum_{\{T\}} C(T) e^{-S}$

where $C(T)$ is a symmetry factor and the sum goes over simplicial complexes $T$. The canonical partition function $Z_{C}$ is defined by an analogous expression, where $N_{4}$ of $T$ is kept fixed. One can investigate the critical behaviour of the theory by studying

$F\left(\kappa_{2}, N_{4}\right)=\log Z_{C}\left(\kappa_{2}, N_{4}\right)$

and its derivatives in the limit $N_{4} \rightarrow \infty$. One finds that the model has two phases. The determination of the exact nature of the transition between these two phases is obviously of utmost importance.

In Ref. [1] it was found, in spherical topology, that the transition is of first order, contrary to the common belief. However, one can wonder

\footnotetext{
*Permanent address: Institute of Physics, Jagellonian University, ul. Reymonta 4, 30-059 Kraków, Poland

†Laboratoire associé au CNRS.
}

whether this finding does not reflect a deficiency of the simulation set-up. As it is an important question, we have decided to check it in more detail. The result of this investigation is presented in sect. 2 of this communication. In sect. 3 we also present some results on the behaviour of the model in other topologies.

\section{The dependence of the transition on the limitations of the algorithm}

In $4 \mathrm{~d}$ there does not exist a set of ergodic local moves preserving the value of $N_{4}$. In order to study the canonical ensemble the standard procedure is to carry out a grand-canonical simulation with a modified action, for example

$S_{\text {mod }}=-\kappa_{2} N_{2}+\kappa_{4}^{0} N_{4}+\frac{\gamma}{2}\left(N_{4}-V_{4}\right)^{2}$

where $V_{4}$ is the value of $N_{4}$ to be studied. All five local moves ergodic in the grand-canonical ensemble are employed. The constant $\kappa_{4}^{0}$ is chosen so that $N_{4}$ fluctuates around $V_{4}: \Delta N_{4}=1 / \sqrt{\gamma}$. Measurements are performed every $n$ 'th time $N_{4}$ crosses $V_{4}$, with an appropriate choice of $n$. In Ref. [1] we have used $\gamma=0.005$ and 0.0005 . The first-order nature of the transition was concluded from finite size scaling and from the appearance of a double-peak structure at $\kappa_{2}=1.258$ for our largest value of $N_{4}=32000$. With $\gamma=0.005$ (0.0005) one has $\Delta N_{4}=14$ (45), a rather modest fluctuation compared to 32000 . However, the constraint limiting volume fluctuations may not be innocuous. Any two lattice configurations at 


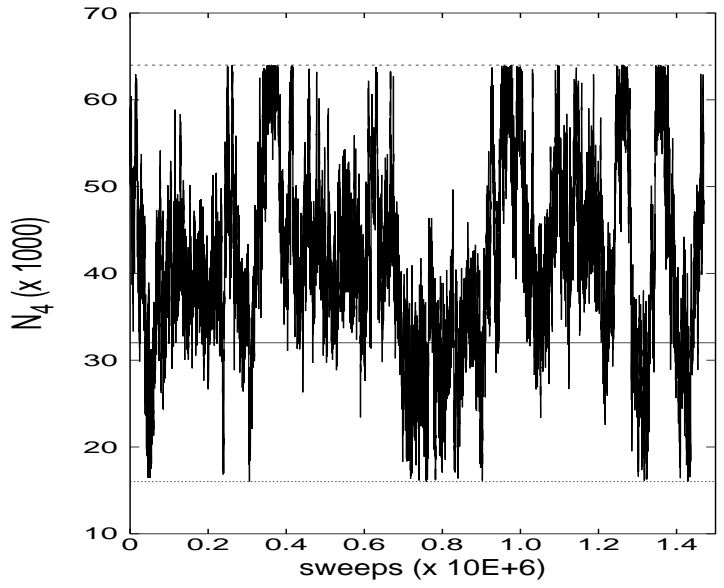

Figure 1. The time history of $N_{4}$.

$N_{4}=V_{4}$ can be connected by a sequence of local moves, provided $N_{4}$ is allowed to deviate enough from $V_{4}$. When this condition is satisfied, the path of the system, in its time evolution and in the space of configurations, recurrently crosses the hyperplane $N_{4}=V_{4}$. This defines the density of states, corresponding to the canonical ensemble. It is not obvious that this density is unaffected when volume fluctuations are constrained. Therefore, we have performed another set of simulations at $\kappa_{2}=1.258$ and $N_{4}=32000$, allowing for fluctuations $\Delta N_{4} \sim 10^{4}$. One cannot just set $\gamma=10^{-8}$ : the finite size corrections to $F\left(\kappa_{2}, N_{4}\right)$ lead to metastable states at different values of $N_{4}$. Thus, instead of (幽 we choose another action

$S_{m o d}=-\kappa_{2} N_{2}+U\left(N_{4}\right)$,

where the external potential $U$ is chosen so as to favour large excursions from the reference volume $V_{4}$. The optimal choice is $U=-F\left(\kappa_{2}, N_{4}\right)$, but the free energy is unknown. Hence, we measured the derivative $\partial F / \partial N_{4}$ for a few volumes, interpolated it linearly and integrated over $N_{4}$. The result can differ within the errors bars from the true free energy and this can make the simulation unstable. To insure stability we added a constant attractive force $\delta=0.0001$ to the derivative and reflecting walls at 16000 and 64000 . The long excursions are evident in the history of $N_{4}$ as shown in Fig.1. We have performed $1.5 \times 10^{6}$ sweeps

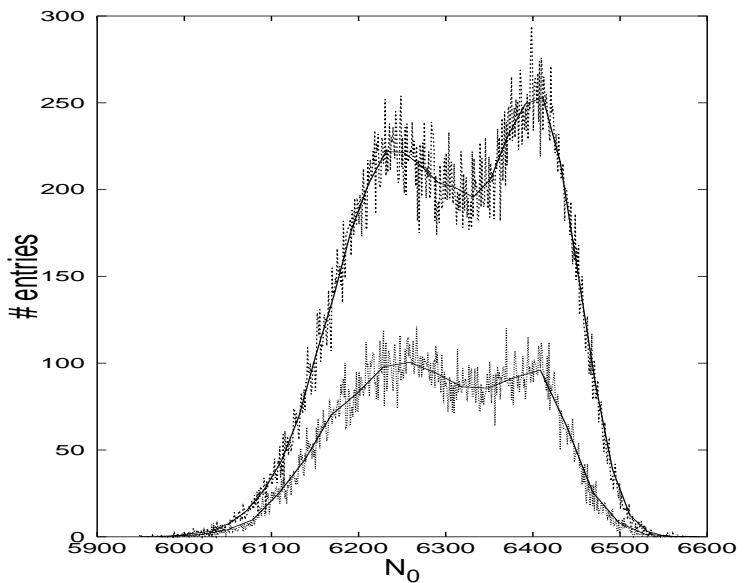

Figure 2. The histograms of $N_{0}$.

and gathered $3 \times 10^{5}$ measurements, which were taken every 100 'th crossing of $V_{4}$. In Fig.2 we show the corresponding $N_{0}$-distribution together with that of Ref. [1]. They are in fact very similar and, in particular, both are bimodal. The first three cumulants of $N_{0}$ distribution are now $0.1966(4), 0.318(26)$ and $-3.0(30)$ to be compared respectively with the values $0.1971(2), 0.316(8)$ and $-6.1(21)$ given in Ref. [1]. They agree within the statistical errors. In Fig.3 we show the history of the average size of minimal neck baby universes, large in the elongated phase and small in the crumpled one, and the order of the most singular point, which has the correlated opposite behaviour. The existence of two states with tunneling between them is very apparent. We conclude that the two state signal, i.e. the first-order nature of the transition is a genuine feature of the model.

\section{Dependence on topology}

We have investigated the dependence of the free energy on topology. More precisely, we have measured

$r=\frac{1}{N_{4}} \frac{\partial F}{\partial \kappa_{2}}=\frac{<N_{2}>}{N_{4}}$

and

$k\left(\kappa_{2}, N_{4}\right)=\frac{\partial F}{\partial N_{4}}$ 


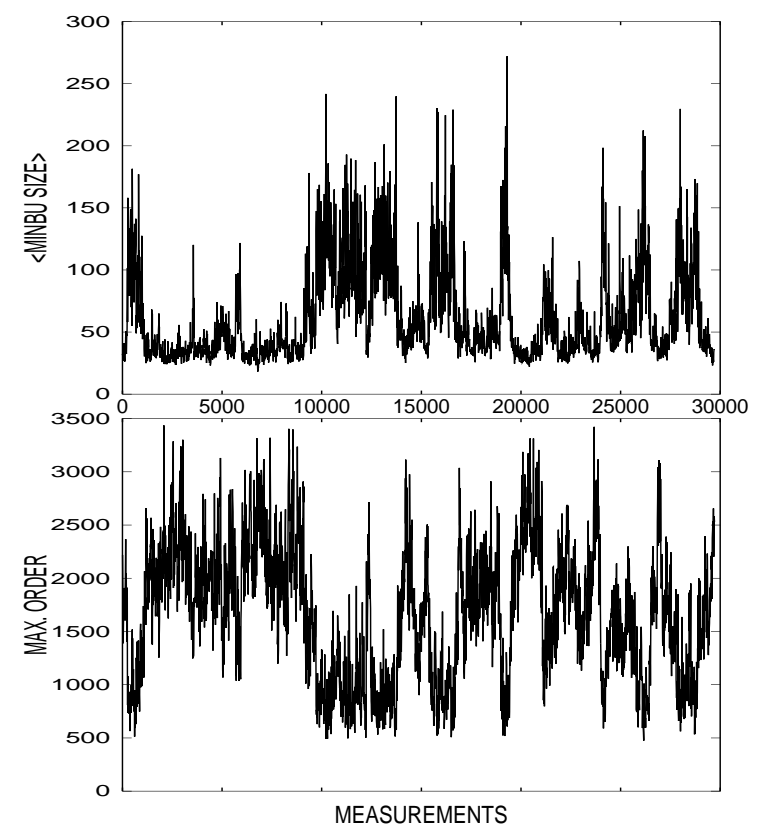

Figure 3. The time history of the maximal vertex order and of the average minbu size.

In the limit $N_{4} \rightarrow \infty$ they become intensive quantities and $k\left(\kappa_{2}, N_{4}\right) \rightarrow \kappa_{4 c r}$. We have simulated the topologies $S^{4}, S^{3} \times S^{1}$ and $\left(S^{1}\right)^{4}$. The minimum configurations have $\left(N_{2 M I N}, N_{4 M I N}\right)$ equal $(20,6),(110,44)$ and $(1472,704)$ respectively. In the elongated phase the most probable move, ie barycentric subdivision, leads to

$$
\begin{aligned}
N_{2} & =\frac{5}{2} N_{4}+c \\
c & =N_{2 M I N}-\frac{5}{2} N_{4 M I N}
\end{aligned}
$$

and $c^{\prime}$ 's are 5,0 and 288 for $S^{4}, S^{3} \times S^{1}$ and $\left(S^{1}\right)^{4}$ respectively. One expects

$$
\begin{aligned}
& r=r_{\infty}+c N_{4}^{-1}+\ldots \\
& k=\kappa_{4 c r}+(\gamma-3) N_{4}^{-1}+\ldots
\end{aligned}
$$

with $\gamma=\frac{1}{2}$. In Fig.4 we show $r$ as a function of $1 / N_{4}$ for $\kappa_{2}=2.0$. In fact, as one can see from figure 4 one gets a very good fit by using $c$ from the minimum configurations.

In the crumpled phase one expects

$$
k=\kappa_{4 c r}+f_{k} N_{4}^{-\delta}+\ldots
$$

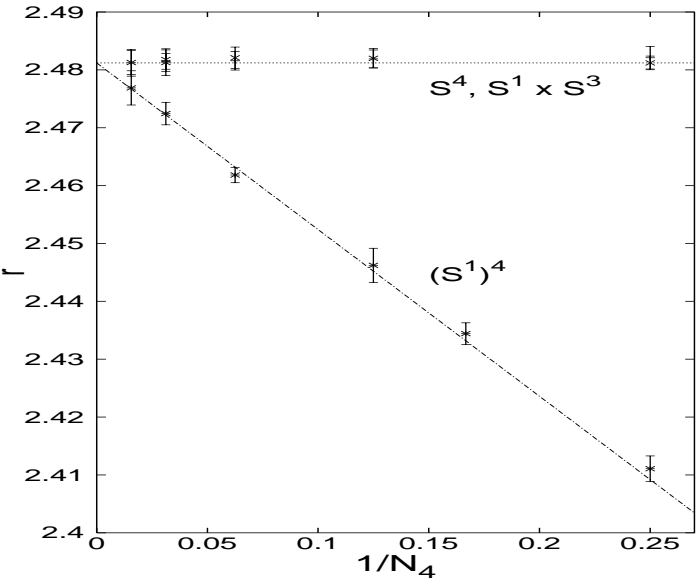

Figure 4. The average action density $r$ vs. system size for various topologies.

$r=r_{\infty}+f_{r} N_{4}^{-\delta}+\ldots$

where the coeeficients $f_{k}, f_{r}$ are functions of $\kappa_{2}$ only. At $\kappa_{2}=0.0$ i.e. in the crumpled phase we have made very extensive runs. We see no dependence on topology there, and the best fit gives $\delta=0.49(12)$. This is in contrast to some earlier results, and consistent with $\delta=1 / 2$. The latter value can be obtained again from a purely kinematical argument, using the fact that the most probable configurations have $N_{4} \sim N_{0}^{2}$. As a conclusion, the termodynamic limit is independent of topology, and the finite size corrections can be understood from purely kinamatic arguments. An extended version of our results on topology dependence will be published in Ref. [2].

We thank the CNRS computing center IDRIS and the HLRZ Jülich for computer time. Z.B. thanks the Deutsche Forschungsgemeinschaft for the financial support under Grant Pe 340/3-3.

\section{REFERENCES}

1. P. Bialas, Z. Burda, A. Krzywicki, B. Petersson, Nucl. Phys. B472 (1996) 293.

2. S. Bilke, Z. Burda, B. Petersson, in preparation. 